\title{
Theoretical study of Interaction between Tacrine and Finite-length Al-doped Carbon and Boron nitride Nanotubes: A Semiempirical Drug Delivery Study in Thermodynamic view
}

\author{
NASRIN ZEIGHAMI*, MOHAMMAD REZA GHOLAMI², \\ MEHRAN AGHAIE ${ }^{3}$ and ASADOLLAH BOSHRA ${ }^{4 *}$ \\ 'Department of Chemistry, Science and Research Branch, Islamic Azad University, Tehran, Iran.
${ }^{2}$ Department of Chemistry, Sharif University of Technology, Tehran, Iran.
${ }^{3}$ Department of Chemistry,North Tehran Branch, Islamic Azad Universitry,Tehran,Iran.
${ }^{4}$ Department of Chemistry, Islamic Azad University, Boroujerd, Iran.
${ }^{*}$ Corresponding author E-mail: n.zeighami@gmail.com, a.boshra @ gmail.com
}

http://dx.doi.org/10.13005/ojc/300441

(Received: September 30, 2014; Accepted: November 03, 2014)

\begin{abstract}
In order to extend our previous theoretical calculations that dealt with the thermochemistry of doping the single walled boron nitride nanotubes, BNNTs, and carbon nanotubes ,CNTs, with alminium atoms [1], we have used the AM1, PM3, and PM6 semiempirical methods to investigate the interaction of the tacrine molecule (a drug for the treatment of Alzheimer's disease) with the side-walls of aluminum doped boron nitride and carbon nanotubes in thermodynamic views. At first, the frequency calculations were carried out to confirm the stability of the involved structures. In addition, the theoretical thermodynamic study of tacrine adsorption onto the considered nanotubes was performed and the thermodynamic functions such as enthalpy changes, entropy changes and Gibbs free energy changes of the adsorption process were evaluated at different temperatures. Our results suggest the aluminum doped boron nitride nanotubes and alminium doped carbon nanotubes may be considered as the proper carries for the drug delivery of tacrine.
\end{abstract}

Key words: Tacrine, Semiempirical methods, Drug delivery, Nanotube, Alzheimer.

\section{INTRODUCTION}

Alzheimer's disease $(A D)$ is a progressive and fatal neurodegenerative disorder manifested by cognitive and memory deterioration, progressive impairment of activities of daily living, and a variety of neuropsychiatric symptoms and behavioral disturbances ${ }^{2}$. Tacrine (Tetrahydroaminoacridine) was the first centrally-acting cholinesterase inhibitor approved for the treatment of Alzheimer's disease ${ }^{3}$. Over the last few years, a variety of biological nanostructures have been synthesized which can 
be utilized for biological therapies, such as drug delivery ${ }^{4}$. Carbon nanotubes (CNTs) have attracted considerable attention due to their unique characteristics as one of the most promising nanostructures for a variety of biomedical applications ${ }^{5}$. They can deliver a range of therapeutic drugs into the cells ${ }^{6-10}$. On the other hand, boron nitride nanotubes (BNNTs) of high purity and quality have advantage over CNTs because they are non-cytotoxic ${ }^{11}$ whereas CNT are quite cytotoxic $^{12-15}$ suggesting BNNT to be suitable for biomedical applications compared to CNT.

Several methods based on semiempirical potentials and particularly the AM1 method ${ }^{16}$ allow to describe correctly the various properties of CNTs ${ }^{17-19}$. Mavrandonakis et al. have studied the interaction of an amino acid with $\mathrm{CNT}^{20}$. Basiuk employed single-level MM+ molecular mechanics and the AM1 semiempirical method to study noncovalent interactions of simple aliphatic amines with carboxylated single-walled carbon nanotube models ${ }^{21}$. Al-anber used PM3 calculations to evaluate the interaction of radical of nitron (anticancer drug) with carbon nanotubes ${ }^{22}$. They studied the effect of the diameter, length, position, and rotation characteristics of the carbon nanotube on binding to the nitron. Al-anber also evaluated the interaction of glycine radicals with carbon SWNTs by MINDO/3 semiempirical method ${ }^{23}$.

In the present study, with the goal of extending the domain of nanotubes specially BNNTs for drug delivery applications, we have carried out a systematic theoretical study on the interaction of tacrine molecule (Figure 1) with the sidewalls of armchair and zigzag nanotubes (Figures 2(a)-2(d)). Actually, we intend to address to the interactions between the stable geometries of tacrine molecule and $(4,4)$ and $(8,0)$ CNTs, and BNNTs sidewalls through the semiempirical thermochemical studies.

\section{Computational Details}

In our previous work, we used the AM1,PM3 and PM6 semiemprical methods for studing the doping of sibgle walled boron nitride nanotubes and carbon nannotubes,BNNTs and CNTs, with aluminium atom and investigating the thermochemistry behavior of the doping reactions and the related thermodynamic properties ${ }^{1}$. There, the results of the AM1,PM3 and PM6 methods showed the same trends for enthalpy changes and Gibbs free energy changes for Al-doping reactions of BNNTs and $(4,4) C N T$. In addition, the AM1 and PM6 levels results revealed that zigzag $(8,0)$ CNT is the best candidate for being doped with aluminium atom at various studied themperatures ${ }^{1}$.

In the present work, we have focused on the tacrine(fig.1) interaction with Al-doped nanotube models (fig.2). This interaction can be showed as the following reaction model:

Al-doped SWNT+Tacrine=Al-doped SWNT..... Tacrine

The necessary theoretical data and the obtimum charactritics for Al-doped SWNTs and tacrine molecule were reported in our previous paper [1].

But at the present work for evaluating the thermodynamic properties of reaction model(1), we examined the"Tacrine...Al-doped SWNTs complex" by means of the same theoretical approach that was used in our previous work[1]. For simplicity,we abbreviate this complex as"T....X" where T stands for Tacrine molecule and $X$ for any considered doped nanotube.

\section{RESULTS AND DISCUSSION}

We used the AM1,PM3 and PM6 semiemperical calculations for deducing the obtimum thermochemistry properties and the other necessary characteristics of"Tacrine...Al-doped SWNT complex ". It is worthy to mention that our calculation showed the undoped SWNTs did not show any tendency for adsorbing tacrine molecules and so, we just considered the Al-doped SWNTs for adsorbing tacrine molecules according to the reaction model.

For evaluating the adsorption of tacrine molecule onto the sidewall of Al-doped SWNTs four configurations were picked up with respect to the four different sites of the tacrine molecule(figure 1). For each site,the related atom of tacrine molecule is pointed toward the doped aluminum atom in the 
middle of nanotube to make a specific configuration Then , each model (Tacrine...NT) was fully optimized by semiemprical methods as mentioned earlier.The distances of the closet atom of adsorbed tacrine molecule onto the Al-doped nanotubes from the Al atom in the optimized models are given in Table3.

In addition the detail results of our theoretical calculations on the basis of various semiemprical methods as us follows:

\section{The AM1 method}

We used the AM1 method to study the"T....X" Systems at different configurations and various temperatures where $T$ denotes Tacrine molecule and $X$ stands for any one of " $\mathrm{Al}(\mathrm{B})$ doped $(4,4) N T$ ","Al(B)-doped $(8,0) B N N T$,"AI(N)doped(8,0)BNNT"," Al-doped(4,4)CNT"

Al-doped(8,0) CNT structures. For simplicity, we abbreviate the mentioned doped nanotubes as $H, K, L, M$ and $Z$ respectively. It is worthwhile to mention that each "T....X" system has four possible configurations respect to four active sites of Tacrine molecule (see fig.1). The results of our calculations revealed that the " $T+X=T . . . X$ " reactions are spontaneous $\left(\Delta \mathrm{G}_{\text {adsorp }<0}\right)$ and most of them exothermic .In figure 3 ,the calculated $\Delta \mathrm{H}_{\text {adsorp }}$ related to the " $\mathrm{T}+\mathrm{X} \rightarrow \mathrm{T}$... $\mathrm{X}$ " process at different temperatures are ploted userus temperature respect to each configuration.

The tacrine adsorption on the surface of $\mathrm{Al}(B)$-doped $(4,4) \mathrm{BNNT}$ is exothermic for various configurations at all applied temperatures.In configurations 1 and 2( Figure 3(a)), tacrine molecule can be adsorbed chemically to the considered nanotube. But, for configurations 3 and 4 of figure 3 the values of enthalpy changes indicate the physisorption process. (As mentioned earlier, for $(8,0) B N N T$, there are two models of Al-doping:(1). when boron atom of tube is replaced with $A$ atom, we obtain $\mathrm{Al}(B)$-doped(8,0)BNNT,(2). and when $B$ atom of tube is replaced with $\mathrm{Al}$ atom,we get $\mathrm{Al}(N)$-doped(8,0)BN).

Table 1: Calculated bond lengths (in $\AA$ ) of nanotubes at different semiempirical methods*

\begin{tabular}{lccc}
\hline \multirow{2}{*}{ Nanotube } & \multicolumn{3}{c}{ (R1, R2) } \\
\cline { 2 - 4 } & AM1 & PM3 & PM6 \\
\hline $\begin{array}{l}\text { (4,4)BNNT } \\
(8,0) B N N T\end{array}$ & $(1.42,1.42)$ & $(1.48,1.47)$ & $(1.47,1.46)$ \\
$(4,4)$ CNT & $(1.41,1.42)$ & $(1.47,1.48)$ & $(1.46,1.47)$ \\
$(8,0) C N T$ & $(1.43,1.43)$ & $(1.44,1.39)$ & $(1.45,1.40)$ \\
& & $(1.43,1.42)$ & $(1.43,1.43)$ \\
\hline
\end{tabular}

For more details see figure 2

Table 2: Calculated Al-N bond lengths (in $\AA$ ) of Al-doped nanotubes at different semiempirical methods

\begin{tabular}{llll}
\hline \multirow{2}{*}{ Doped Nanotube } & \multicolumn{3}{c}{ Al-N bond length } \\
\cline { 2 - 4 } & AM1 & PM3 & PM6 \\
\hline Al(B)-doped(4,4)BNNT & 1.69 & 1.77 & 1.78 \\
Al(B)-doped(8,0)BNNT & 1.68 & 1.76 & 1.77 \\
Al(N)-doped(8,0)BNNT & 1.78 & 1.77 & 1.95 \\
Al-(4,4)CNT & 1.73 & 1.76 & 1.88 \\
Al- $(8,0) C N T$ & 1.71 & 1.75 & 1.88 \\
& & & \\
\hline
\end{tabular}

${ }^{\star}$ For more details see Figure 2
The results for $\mathrm{Al}(B)$-doped $(8,0) \mathrm{BNNT}$ (Figure $3(b)$ ) had the same trend showing that the adsorption is as $\mathrm{Al}(B)$-doped $(4,4) B N N T$. All configurations showed exothermic adsorptions: Chemisorption for the configurations 1 and 2, and physisorption for configurations 3 and 4 . In the case of $\mathrm{Al}(N)$-doped $(8,0) B N N T$, the process was chemisorption for all configurations in all temperatures (Table 3).

Tacrine adsorption on the $\mathrm{Al}$ doped(4,4)CNT (Figure 3(d)) showed both exothermic and endothermic process depending on the considered configuration. Configurations 1 , 


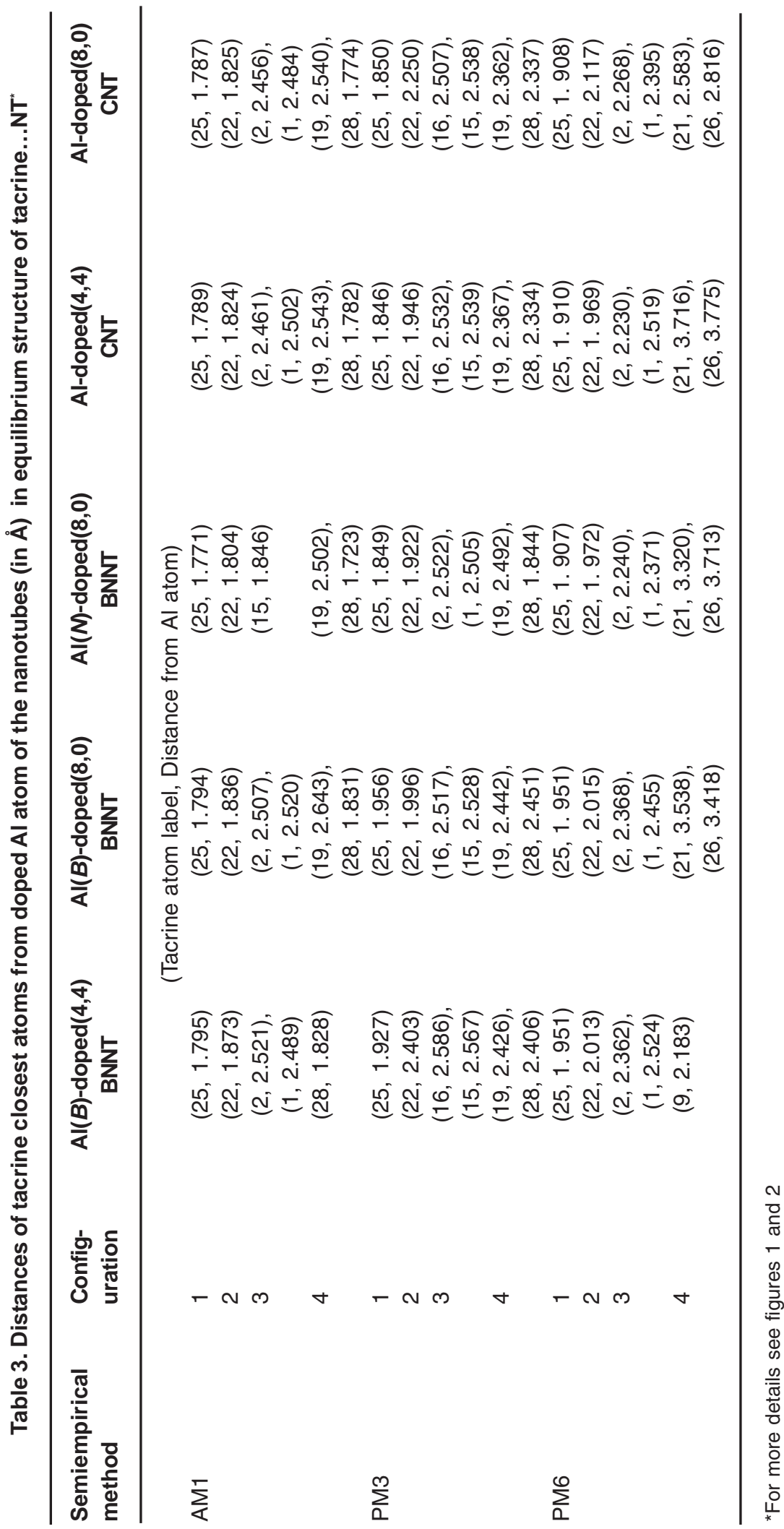


2, and, 3 indicated exothermic chemisorption while configuration 4 was endothermic and nonspontaneous. The calculated results showed that the Al-doped $(4,4) \mathrm{CNT}$ is more favorable than BNNTs for Tacrine adsorption. The Tacrine adsorption onto the $\mathrm{Al}$-doped $(8,0) \mathrm{CNT}$ was spontaneous and highly exothermic-chemisorption for all configurations.

The PM3 method

The enthalpy changes for the process"

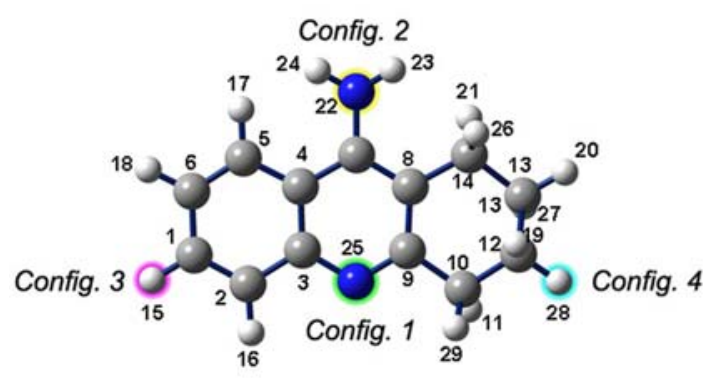

Fig. 1: The optimized structure of tactine molecule.

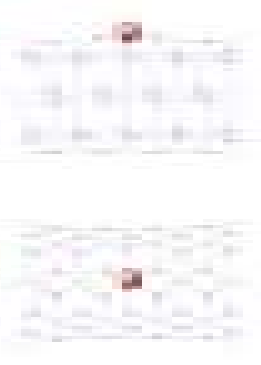

$=$
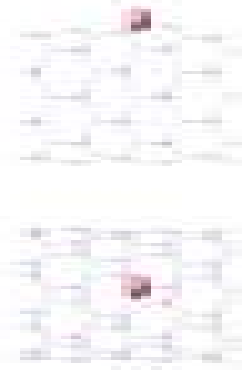

-

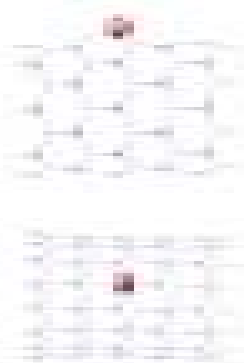

$=$
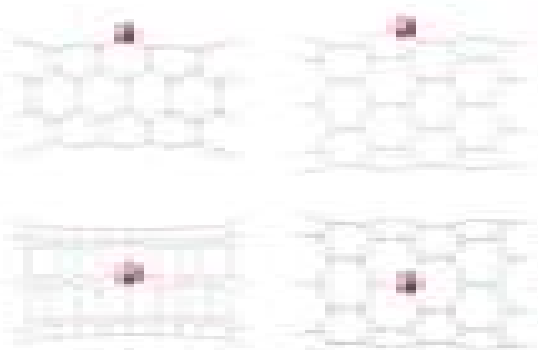

H

Fig. 2: The optimized structures of (a) Al(B)-doped $(4,4) B N N T$ (b) Al(B)-doped $(8,0)$ BNNT (c) Al(N)-doped (8,0)BNNT (d) Al-doped $(4,4)$ CNT (e) Al-doped $(8,0) C N T$
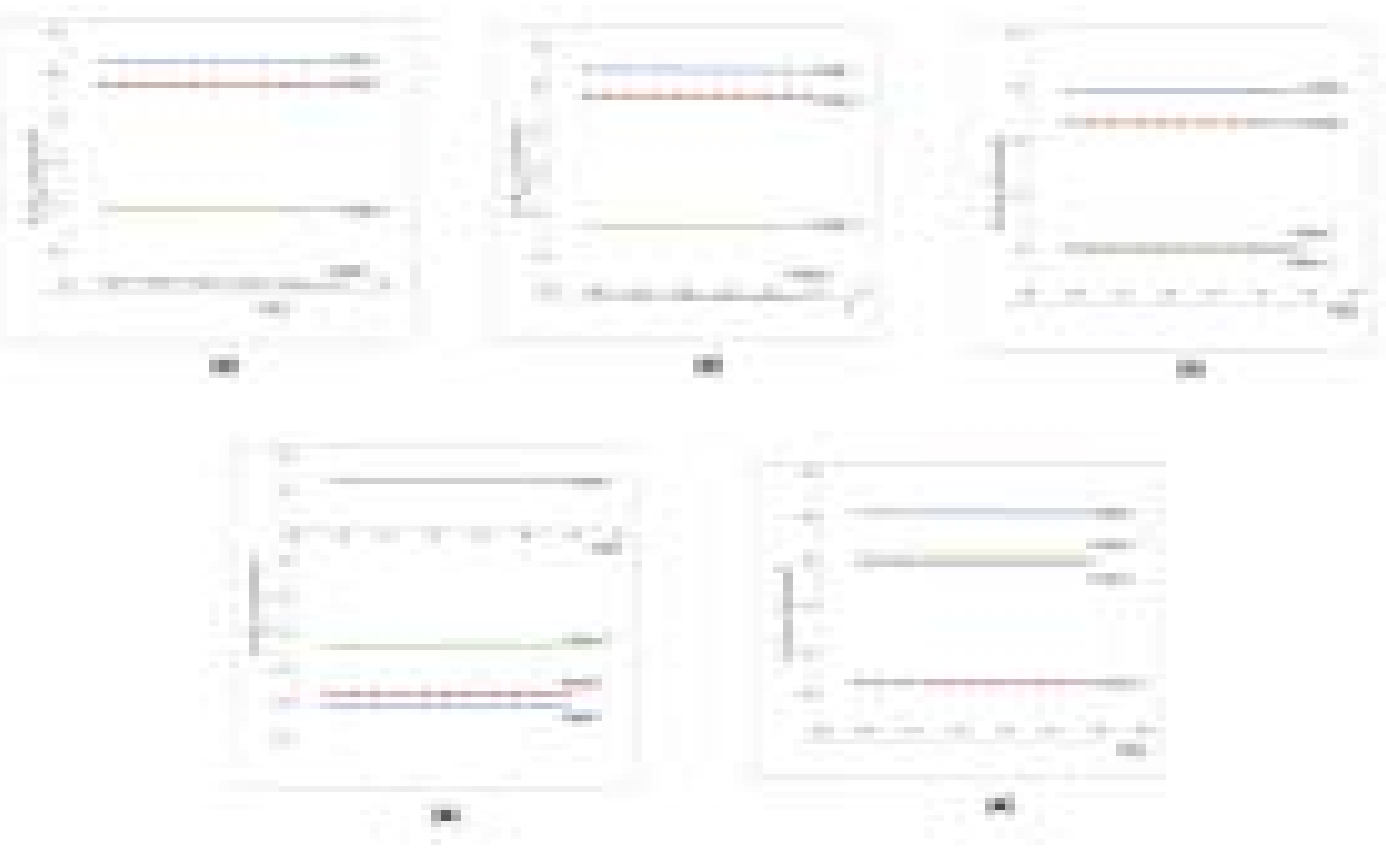

Fig. 3. $\Delta_{\mathrm{r}} \mathrm{H}_{\mathrm{ads}}$ curves of different configurations of tacrine molecule on (a) $\mathrm{Al}(\mathrm{B})$-doped $(4,4) B N N T$ (b) Al(B)-doped $(8,0) B N N T$ (c) Al( $N)$-doped $(8,0) B N N T$ (d) Al-doped $(4,4)$ CNT (e) Al-doped $(8,0)$ CNT at AM1 semiempirical levels of theory 


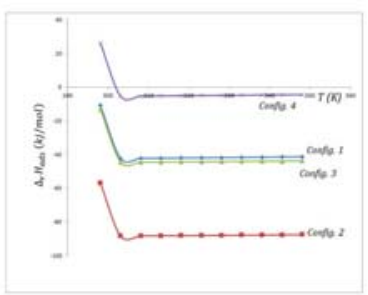

(a)

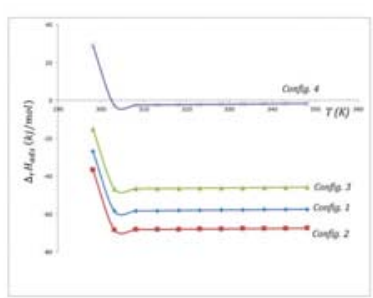

(b)

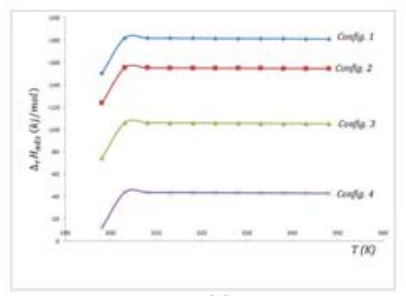

(c)

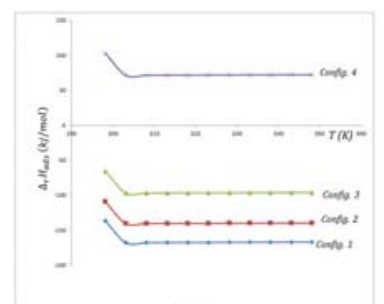

(d)

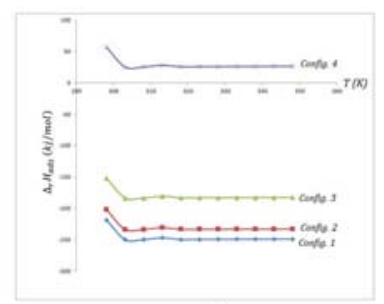

(e)

Fig. 4: $\Delta_{\mathrm{r}} \mathrm{H}_{\text {ads }}$ curves of different configurations of tacrine on (a) Al(B)-doped (4,4)BNNT (b) Al(B)-doped (8,0)BNNT (c) Al(N)-doped $(8,0) B N N T$ (d) Al-doped $(4,4) C N T$ (e) Al-doped $(8,0)$ CNT at PM3 semiempirical levels of theory

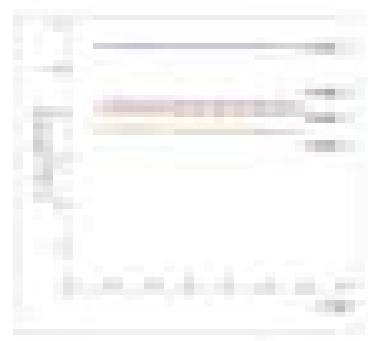

플
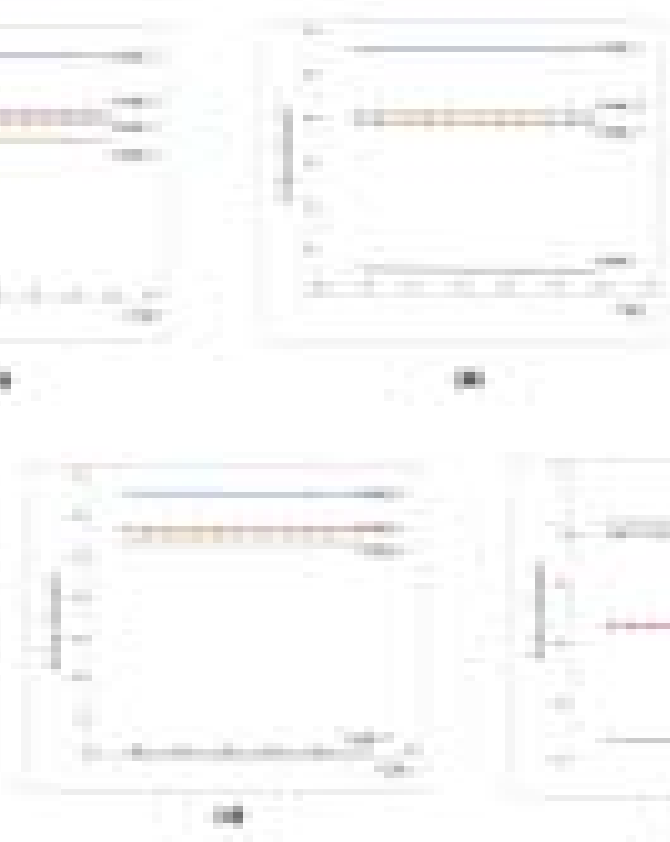

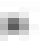

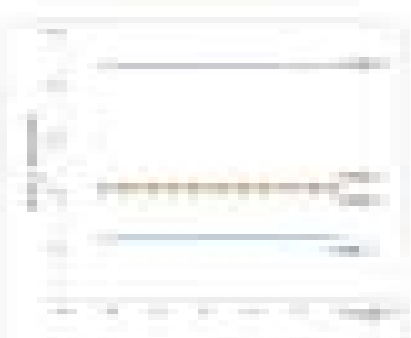

Fig. 5: $\Delta_{\mathrm{r}} \mathrm{H}_{\text {ads }}$ curves of different configurations of tacrine on (a) Al(B)-doped $(4,4) B N N T$ (b) Al(B)-doped (8,0)BNNT (c) Al(N)-doped (8,0)BNNT (d) Al-doped $(4,4) C N T$ (e) Al-doped $(8,0)$ CNT at PM6 semiempirical levels of theory 
$\mathrm{T}+\mathrm{X}=\mathrm{T} . . . \mathrm{X}$ " were evaluated upon the PM3 method at different temperatures for all $\mathrm{H}, \mathrm{K}, \mathrm{L}, \mathrm{M}$, and $\mathrm{Z}$ structures and are ploted in figure $4(a, b, c, d)$. The trend of $\Delta \mathrm{H}_{\text {adsorp }}$ changes with temperature for tacrine adsorption on to the $\mathrm{Al}(\mathrm{B})$-doped $(4,4) \mathrm{BNNT}$ structure was as AM1 except at 298.15K (see figure 4(a)). The PM3 method prediction showed Tacrine adsorption. on to the $\mathrm{Al}(\mathrm{B})$-doped(8,0)BNNT is physisorption (figure4(b)).In addition,the Tacrine adsorption onto the $\mathrm{Al}(\mathrm{N})$-doped(8,0)BNNT showed to be chemisorptions at temperatures higher han 298.15K (figure4(C)). As can be seen from figure $4(\mathrm{~d}$ and $\mathrm{e})$, the " $\mathrm{T}+\mathrm{X}=\mathrm{T}$...X" adsorption may be assumed chemisorption respect to the 1,2 and 3 configurations, but $\Delta \mathrm{H}_{\text {adsorp }}$ is positive respect to the configuration1. It seems, the Tacrine adsoption onto the carbone nanotubes is more favorable than boron-nitride nanotubes.

\section{The PM6 method}

As before, the " $\mathrm{T}+\mathrm{X} \vDash \mathrm{E} ! \mathrm{T} . . \mathrm{X}$ " adsorption were investigated upon the PM6 method. The results revealed that all configurations of the "Tacrine..Al(B)-doped (4,4)BNNT" adsorptions may be assumed chemisorptions with covalent bond formation between Tacrine molecule and Al doped atom(figure 5(a)). Indeed, the " $\mathrm{H}_{\text {adsorp }}$ for the considered adsorptions are so negative that one can assume the chemsorption for the considered adsorptions.

The magnitude of " $\mathrm{H}_{\text {adsorp }}$ for the "Tacrine ... $\mathrm{Al}(\mathrm{B})$ doped $(8,0)$ BNNT. system respect to the configuration $5(\mathrm{~b})$, indicated that it may be a physisorption process(see figure 5(b)), but respect to the other configurations are so negative that one can considered them as chemisorptions phenomena.(see figure $5(b)$ ).
The Tacrine adsorption onto the " $\mathrm{Al}(\mathrm{N})$ doped $(8,0)$ BNNT structure, for all configurations were endothermic and nonspontaneous (figure 5(c)).

As shown in figure5(d), Tacrine molecule can be chemically adsorbed in configurations 1,2 and 3 onto the Al-doped $(4,4) \mathrm{CNT}$ with considerable negative enthalpies of adsorption, But the enthalpy change respect to the configuration 4 indicated it is as a physisorption process.

For the Al-doped $(8,0) \mathrm{CNT}$, the tacrine adsorption occurs chemically at configuration 1,2, physisorption at 4 and endothermic at configuration $5(e)$ (see figures $(3 e, 4 e, 5 e)$.

\section{CONCLUSION}

We theoretically investigated the interaction of tacrine molecule (a drug for the treatment of Alzheimer's disease) with finite-length aluminum doped armchair and zigzag boron nitride, and carbon nanotubes. All nanotubes were chosen to be single-walled and finite length with hydrogen saturation in both ends. The semiempirical quantum chemistry techniques AM1, PM3, and PM6 were employed for the study. The obtained thermochemical properties based on the mentioned semiempirical methods predicted chemisorption for most of cases and in a fiew cases physisorption of tacrine on the sidewall of Al-doped nanotubes.Furthermore,the armchair and zigzag carbon nanotubes were more effective for tacrin molecules adsorbing onto their surfaces than boron nitride counterparts. The enthalpy changes of the considered adsorption phenomena showed that Al-doped armchair and zigzag nanotubes might be suitable as nanocarriers for tacrine delivery of Alzheimer's drug.

\section{REFERENCES}

1. Zeighami, N., Boshra, A., Gholami, M.R., Aghaie,M.J.Phys. Theor.Chem.IAU.Iran, spring 2014, 11(1), 47-53

2. Brookmeyer, R., Johnson, E., ZieglerGraham, K., Arrighi, H.M. Forecasting the global burden of Alzheimer's disease.
Alzheimer's \& dementia. 2007, 3, 186-91.

3. Taraschenko, O., Barnes, W., Herrick-Davis, K., Yokoyama, Y., Boyd, D., Hough, L. Actions of tacrine and galanthamine on histamine$\mathrm{N}$-methyltransferase. Methods and findings in experimental and clinical pharmacology. 
2005, 27, 161-6.

4. Ghanbari, H., de Mel, A., Seifalian, A.M. Cardiovascular application of polyhedral oligomeric silsesquioxane nanomaterials: a glimpse into prospective horizons. International journal of nanomedicine. 2011, $6,775$.

5. Sahoo, N.G., Bao, H., Pan, Y., Pal, M., Kakran, M., Cheng, H.K.F., et al. Functionalized carbon nanomaterials as nanocarriers for loading and delivery of a poorly watersoluble anticancer drug: a comparative study. Chemical Communications. 2011, 47, 52357.

6. Pantarotto, D., Singh, R., McCarthy, D., Erhardt, M., Briand, J.P., Prato, M., et al. Functionalized carbon nanotubes for plasmid DNA gene delivery. Angewandte Chemie. 2004, 116, 5354-8.

7. Cai, D., Mataraza, J.M., Qin, Z.-H., Huang, Z., Huang, J., Chiles, T.C., et al. Highly efficient molecular delivery into mammalian cells using carbon nanotube spearing. Nature Methods. 2005, 2, 449-54.

8. Gao, L., Nie, L., Wang, T., Qin, Y., Guo, Z., Yang, D., et al. Carbon nanotube delivery of the GFP gene into mammalian cells. ChemBioChem. 2006, 7, 239-42.

9. Kam, N.W.S., Dai, H. Carbon nanotubes as intracellular protein transporters: generality and biological functionality. Journal of the American Chemical Society. 2005, 127, 6021-6.

10. Cherukuri, P., Bachilo, S.M., Litovsky, S.H., Weisman, R.B. Near-infrared fluorescence microscopy of single-walled carbon nanotubes in phagocytic cells. Journal of the American Chemical Society. 2004, 126, 15638-9.

11. Chen, X., Wu, P., Rousseas, M., Okawa, D., Gartner, Z., Zettl, A., et al. Boron nitride nanotubes are noncytotoxic and can be functionalized for interaction with proteins and cells. Journal of the American Chemical Society. 2009, 131, 890-1.

12. Rouse, J.G., Yang, J., Barron, A.R., MonteiroRiviere, N.A. Fullerene-based amino acid nanoparticle interactions with human epidermal keratinocytes. Toxicology in vitro. 2006, 20, 1313-20.
13. Monteiro-Riviere, N.A., Nemanich, R.J., Inman, A.O., Wang, Y.Y., Riviere, J.E. Multiwalled carbon nanotube interactions with human epidermal keratinocytes. Toxicology letters. 2005, 155, 377-84.

14. Shvedova, A., Castranova, V., Kisin, E., Schwegler-Berry, D., Murray, A., Gandelsman, V., et al. Exposure to carbon nanotube material: assessment of nanotube cytotoxicity using human keratinocyte cells. Journal of toxicology and environmental health Part A. 2003, 66, 1909-26.

15. Bottini, M., Bruckner, S., Nika, K., Bottini, N., Bellucci, S., Magrini, A., et al. Multi-walled carbon nanotubes induce $\mathrm{T}$ lymphocyte apoptosis. Toxicology letters. 2006, 160, 1216.

16. Dewar, M.J., Zoebisch, E.G., Healy, E.F., Stewart, J.J. Development and use of quantum mechanical molecular models. 76 . AM1: a new general purpose quantum mechanical molecular model. Journal of the American Chemical Society. 1985, 107, 3902-9.

17. Ormsby, J.L., King, B.T. The regioselectivity of addition to carbon nanotube segments. The Journal of organic chemistry. 2007, 72, 4035-8.

18. Rochefort, A., Salahub, D.R., Avouris, P. Effects of finite length on the electronic structure of carbon nanotubes. The Journal of Physical Chemistry B. 1999, 103, 641-6.

19. Türker, L., Erkoç, ^Õ. Electronic properties of open-ended single wall carbon nanotubes. Journal of Molecular Structure:THEOCHEM. 2002, 577, 131-5.

20. Mavrandonakis, A., Farantos, S.C., Froudakis, G.E. Theoretical modeling of glycine radical addition to carbon nanotubes. Rev. Adv. Mater. Sci. 2006, 11, 5.

21. Basiuk, V.A. ONIOM studies of chemical reactions on carbon nanotube tips: effects of the lower theoretical level and mutual orientation of the reactants. The Journal of Physical Chemistry B. 2003, 107, 8890-7.

22. Al-anber, M.J., Ali, A.M., Resan, S.F., Almouali, A.H. The Nitron (Anti-cancer drug) Interaction with Carbon Nanotubes (Delivery): The Semi-Empirical Approach. International Journal of Green 
Nanotechnology. 2011, 3, 238-43.

23. Al-Anber, M.J. Theoretical Semiempirical Study of the Biomolecules Interaction with Carbon Nanotubes. Journal of Macromolecular Science, Part B. 2011, 50, 2481-7.

24. Majidi, R., Ghafoori Tabrizi, K., Jalili, S. Effect of doping on electronic properties of doublewalled carbon and boron nitride heteronanotubes. Physica B: Condensed Matter. 2009, 404, 3417-20.

25. Cioslowski, J., Rao, N., Moncrieff, D. Electronic structures and energetics of $[5,5]$ and $[9,0]$ single-walled carbon nanotubes. Journal of the American Chemical Society. 2002, 124, 8485-9.

26. Mercuri, F., Sgamellotti, A. DFT methods in the chemistry of carbon nanotubes. In: Chemistry of carbon nanotubes, Basiuk V. Basiuk, E. Eds., American Scientific Publishers, 2007.

27. Dewar, M.J., Thiel, W. Ground states of molecules. 38. The MNDO method. Approximations and parameters. Journal of the American Chemical Society. 1977, 99, 4899-907.

28. Stewart, J.J. Optimization of parameters for semiempirical methods I. Method. Journal of Computational Chemistry. 1989, 10, 209-20.

29. Stewart, J.J. Optimization of parameters for semiempirical methods $\mathrm{V}$ : modification of NDDO approximations and application to 70 elements. Journal of Molecular modeling. 2007, 13, 1173-213.

30. JJP, S. MOPAC2012, Stewart computational chemistry. Colorado Springs, CO.

31. Zhou, Z., Zhao, J., Chen, Z., Schleyer, P.v.R. Atomic and electronic structures of fluorinated BN nanotubes: Computational study. The Journal of Physical Chemistry $B$. 2006, 110, 25678-85.

32. Saito, R., Dresselhaus, G., Dresselhaus, M. Physical Properties of Carbon Nanotubes, Imperial College Press: London, 2001. 